Publicacions Matemàtiques, Vol 38 (1994), 441-454.

\title{
MULTIPLIER EXTENSION AND SAMPLING THEOREM ON HARDY SPACES
}

\author{
QIYU SUN
}

\begin{abstract}
Extension by integer translates of compactly supported function for multiplier spaces on periodic Hardy spaces to multiplier spaces on Hardy spaces is given. Shannon sampling theorem is extended to Hardy spaces.
\end{abstract}

\section{Introduction and statement of results}

The purpose of this paper is to establish a natural extension from multiplier spaces $\tilde{M}(p)$ on periodic Hardy spaces $H^{p}(T)$ to multiplier spaces $M(p)$ on Hardy spaces $H^{p}(R)$ by integer translates of a function $\phi$ and to extend Shannon sampling theorem to Hardy spaces. It is the continuation of [13] on stability of integer translates of a function but with different interest. In [13], the following stability problem of integer translates of $\phi$

$$
C^{-1}\|f\|_{H^{p}(Z)} \leq\left\|\sum_{n \in \mathbb{Z}} f(n) \phi(x-n)\right\|_{H^{p}(R)} \leq C\|f\|_{H^{p}(Z)}
$$

was considered which arises in the interpolation of sequences by functions and plays an important role in multiresolution analysis, where $0<p<$ $\infty, f=\{f(n)\}_{n \in Z}$ is a tempered sequence, $H^{p}(Z)$ and $H^{p}(R)$ denotes Hardy spaces on $Z$ and $R$ respectively, and $Z$ is the set of integers. A natural replacement of the norm in (1) when $p=\infty$ is the norm as multiplier operator on $H^{p}(T)$ and $H^{p}(R)$ respectively, which is an

This project is partially supported by Postdoctoral Fellowship Foundation of China and Natural Sciences Foundation of China. 
original inspiration to consider multiplier extension here. To this end, we introduce some notations.

Let $\Phi$ be a smooth function such that supp $\hat{\Phi} \subset\left\{\frac{15}{40} \leq|x| \leq \frac{9}{10}\right\}$, $|\hat{\Phi}(x)| \geq C_{0}$ on $\left\{\frac{7}{20} \leq|x| \leq \frac{4}{5}\right\}$ and $\sum_{m \in Z} \hat{\Phi}\left(2^{m} x\right)=1$ for $x \neq 0$, where $C_{0}$ is a positive constant and the Fourier transform is defined by $\hat{\Phi}(x)=$ $\int e^{2 \pi i x y} \Phi(y) d y$. Denote $\Phi_{m}(x)=2^{m} \Phi\left(2^{m} x\right)$ for $m \in Z$. Now we define Hardy spaces $H^{p}(R)$ by

$$
H^{p}(R)=\left\{f \in S^{\prime}(R) ;\|f\|_{H^{p}(R)}=\left\|\left(\sum_{m \in Z}\left|\Phi_{m} * f\right|^{2}\right)^{\frac{1}{2}}\right\|_{L_{p}(R)}<+\infty\right\}
$$

and define Hardy spaces $H^{p}(T)$ (c.f. [3]) by

$$
\begin{aligned}
H^{p}(T)=\left\{f(x)=\sum_{k \in Z} f_{k} e^{2 \pi i k x} \in S^{\prime}(R) ;\right. & \left.\left|f_{0}\right|+\left\|\left(\sum_{m \geq-1}\left|\Phi_{m} * f\right|^{2}\right)^{\frac{1}{2}}\right\| \|_{L^{p}(T)}<+\infty\right\} \\
= & \left\{\begin{array}{c}
f(x)=\sum_{k \in Z} f_{k} e^{2 \pi i k x} \in S^{\prime}(R) ; \\
\left.\|f\|_{H^{p}(T)}=\left|f_{0}\right|+\mid \int_{0}^{1}\left(\left.\sum_{m \geq 0}\left|\sum_{\substack{2^{m} \leq|k| \\
<2^{m+1}}} f_{k} e^{2 \pi i k x}\right|^{2}\right|^{\frac{p}{2}} d x\right)^{\frac{1}{p}}<+\infty\right\},
\end{array}\right.
\end{aligned}
$$

where we denote the space of tempered distributions by $S^{\prime}(R)$, the norm of $p$-integrable functions on $R$ and $T$ by $\|\cdot\|_{L^{p}(R)}$ and by $\|\cdot\|_{L^{p}(T)}$, respectively and $T=R / Z$ denotes the torus. For a measurable function $m$ on $R$, we say that $m$ is a multiplier on $H^{p}(R)$ if

$$
\|F\|_{H^{p}(R)} \leq C_{m}\|f\|_{H^{p}(R)}
$$

holds for any Schwartz function $f$, where $\hat{F}=m \hat{f}$. We denote the infinum $C_{m}$ in (2) by $\|m\|_{M(p)}$. For a sequence $\tilde{m}=\{\tilde{m}(n)\}$, we say that $\tilde{m}$ is a multiplier on $H^{p}(T)$ if

$$
\|G\|_{H^{p}(T)} \leq C_{\tilde{m}}\|g\|_{H^{p}(T)}
$$


holds for every trigonometric polynomial $g(x)=\sum_{k \in Z} g_{k} e^{2 \pi i k x}$, where $G(x)=\sum_{k \in Z} \tilde{m}(k) g_{k} e^{2 \pi i k x}$. Also we denote $\|\tilde{m}\|_{\tilde{M}(p)}$ the infinum $C_{\tilde{m}}$ in (3).

The classical result of de Leeuw [7] on multiplier said the restriction to the integer lattice of a continuous multiplier on $L^{p}(R)$ is a multiplier on $L^{p}(T)$ when $1<p<\infty$. In 1992, Liu [8] extended the above conclusion to Hardy spaces. The multiplier extension was considered by Jodeit [6], Berkson and Gillespie [2]. Let $\phi$ be a continuous function with compact support. Denote the space of sequences by $S$ and the linear span of integer translates of $\phi$ by $S(\phi)=\left\{\sum_{n \in Z} C(n) \phi(x-n) ;\{C(n)\} \in S\right\}$. Define a natural map $\phi *^{\prime}$ from $S$ to $S(\phi)$ by

$$
\phi *^{\prime}: S \ni\{C(n)\} \longmapsto \sum_{n \in Z} C(n) \phi(x-n) \in S(\phi) .
$$

We say that the integer translates of $\phi$ are globally linearly independent if $\phi *^{\prime}$ is one-to-one. Denote the restriction of $\phi *^{\prime}$ on $\tilde{M}(p)$ by $I$. Berkson and Gillespie [2] proved that $I$ maps $\tilde{M}(p)$ to $M(p)$ boundedly under the hypotheses $1<p<\infty$ and $\phi=\chi_{\left[-\frac{1}{2}, \frac{1}{2}\right]} * \Lambda_{0}$, where $\chi_{\left[-\frac{1}{2}, \frac{1}{2}\right]}$ is the characteristic function of $\left[-\frac{1}{2}, \frac{1}{2}\right], *$ denotes the convolution operator and $\Lambda_{0}$ is a bounded variation function supported in $\left[-\frac{1}{2}, \frac{1}{2}\right]$. In this paper, we will prove

Theorem 1. Let $0<p<\infty$ and $\phi$ have compact support. If $\int|\hat{\phi}(x)|^{\min (1, p)} d x<+\infty$, then I maps $\tilde{M}(p)$ to $M(p)$ boundedly.

We improve Berkson and Gillespie's result since under their hypotheses $|\hat{\phi}(x)| \leq C(1+|x|)^{-2}$ and $\int|\hat{\phi}(x)| d x<\infty$. Applying to Bochner-Riesz summation operator $B_{\delta}$, we reproved that $B_{\delta}$ maps $H^{p}\left(R^{n}\right)$ to $H^{p}\left(R^{n}\right)$ when $\delta>\frac{n}{p}-\frac{n+1}{2}$ and $0<p \leq 1[\mathbf{1 1}],[\mathbf{1 2}]$ when we let $\phi(x)=\left(1-|x|^{2}\right)_{+}^{\delta}$.

To consider the inverse of Theorem 1, we introduce the paraproduct $P_{h}$ and show that $P_{h}$ maps $H^{p}(T)$ to $H^{p}(R)$.

Theorem 2. Let $\phi$ be a continuous function with compact support. If $\phi *^{\prime}: S \rightarrow S(\phi)$ is one-to-one, then $I$ has bounded inverse $I^{-1}: M(p) \cap$ $S(\phi) \rightarrow \tilde{M}(p)$. 
In the proof of Theorem 1 and Theorem 2, Lemma 2 plays an important role. If we assume Lemma 2 is true, or $m(x)=\sum_{n \in Z} C(n) \phi(x-n) \in$ $L^{\infty}$ implies $\{C(n)\} \in l^{\infty}$, then it suffices to assume $I$ is one-to-one in Theorem 2. In particular Theorem 2 can be written as that $I$ has bounded inverse $I^{-1}: M(p) \cap S(\phi) \rightarrow \tilde{M}(p)$ provided $I$ has bounded inverse $I^{-1}: M(2) \cap S(\phi) \rightarrow \tilde{M}(2)$ and $\phi$ is a continuous function with compact support, where $0<p<\infty$. The continuity condition on $\phi$ can be dropped in one spatial dimension since for any distribution $\phi$ on $R$ such that $\phi *^{\prime}$ is one-to-one there exists a univariate spline $B_{k}$ such that $\psi=B_{k} * \phi$ is continuous and $\psi *^{\prime}$ is one-to-one. But I do not know how to construct this modifier $B_{k}$ in high spatial dimensions. By Fourier transform characterization of global linear independence in [9], the box spline and Daubechies' scaling function satisfy the condition on $\phi$ in Theorem 2.

Shannon sampling theorem [10] plays an important role in signal analysis. It says a function with its Fourier transform supported in $\left[-\frac{1}{2}+\varepsilon, \frac{1}{2}-\varepsilon\right]$ for some $0<\varepsilon<\frac{1}{2}$ has its $L^{p}(R)$ norm comparable to its $l^{p}(Z)$ norm of its restriction to integer lattices $Z$ where $1<$ $p<\infty$. In 1990, R. Torres [14] extended the above conclusion to Besov spaces. Let $\left\{\tilde{\Phi}_{m}\right\}_{m \geq 0}$ be a family of sequences such that $\hat{\tilde{\Phi}}_{m}$ is smooth, $\operatorname{supp} \hat{\tilde{\Phi}}_{m}(\xi) \subset\left\{2^{-m-2} \leq|\xi| \leq 2^{-m}\right\},\left|\hat{\tilde{\Phi}}_{m}(\xi)\right| \geq C_{0}$ on $T_{m}$, where $\hat{\tilde{\Phi}}_{m}(\xi)=\sum_{n \in Z} \tilde{\Phi}_{m}(n) e^{2 \pi i n \xi}, T_{0}=\left\{\frac{3}{8} \leq|\xi| \leq 1\right\}$ and $T_{m}=$ $\left\{\frac{3}{8} 2^{-m} \leq|\xi| \leq \frac{9}{10} 2^{-m}\right\}$. Define (c.f. [13] or [14])

$$
H^{p}(Z)=\left\{\{f(n)\}_{n \in Z} ;\left(\sum_{n \in Z}\left(\sum_{m \geq 0}\left|\tilde{\Phi}_{m} * f(n)\right|^{2}\right)^{\frac{p}{2}}\right)^{\frac{1}{p}}<+\infty\right\}
$$

where $f=\{f(n)\}$ is a tempered sequence and $\tilde{\Phi}_{m} * f(n)=\sum_{k \in Z} \tilde{\Phi}_{m}(n-$ $k) f(k)$.

Theorem 3. Let $0<p<+\infty$. If $f \in S^{\prime}(R)$ with supp $\hat{f} \subset$ $\left[-\frac{1}{2}+\varepsilon, \frac{1}{2}-\varepsilon\right]$ for some $0<\varepsilon<\frac{1}{2}$, then the inequality

$$
C^{-1}\|\{f(n)\}\|_{H^{p}(Z)} \leq\|f\|_{H^{p}(R)} \leq C\|\{f(n)\}\|_{H^{p}(Z)}
$$

holds for some constant $C$ dependent of $\epsilon$ and $p$ only. 
For simplicity in the exposition we restrict ourselves to one spatial dimension, all results can be extended to high spatial dimensions. The results of Theorem 1 and 3 can be extended to spaces of Triebel-Lizorkin type trivially. The big letter $C$ will denote different constant at different occurance.

\section{Some lemmas}

To prove our theorems, we will use the following fundamental lemmas.

Lemma 1. c.f. [15]. Let $f \in S^{\prime}(R)$ have its Fourier transform contained in a compact set. Therefore

$$
|\psi * f(x)| \leq C M\left(|f|^{r}\right)^{\frac{1}{r}}(x)
$$

holds for every Schwartz function $\psi$ and $0<r<1$, where $M$ denotes Hardy-Littlewood maximal operator and the constant $C$ depends on the seminorm of $\psi, r$ and the radius $R$ for which $\hat{f}$ is supported in the ball with radius $R$ and center zero.

Proof of Lemma 1: Without loss of generality we assume $x=0$, $M\left(|f|^{r}\right)(0)<+\infty$ and supp $\hat{f} \subset\left[-\frac{1}{4}, \frac{1}{4}\right]$ by dilation invariance. Write

$$
f(x)=\sum_{n \in Z} f(n) \varphi(x-n),
$$

for some Schwartz function $\varphi$ such that $\operatorname{supp} \hat{\varphi} \subset\left[-\frac{3}{8}, \frac{3}{8}\right]$. Hence $\mid f *$ $\left.\psi(0)\right|^{r} \leq \sum_{n \in Z}|f(n)|^{r}(1+|n|)^{-3}$. To prove (4), we first prove $\sum_{n \in Z}|f(n)|^{r}(1+|n|)^{-3}<+\infty$. Recall that $f \in S^{\prime}(R)$ and supp $\hat{f} \subset$ $\left[-\frac{1}{4}, \frac{1}{4}\right]$. Therefore $|f(n)| \leq C(1+|n|)^{N}$ for some constants $C$ and $N$. On the other hand we have

$$
|f(n)|^{r} \leq|f(n+\delta)|^{r}+C|\delta|^{r} \sum_{m \in Z}|f(m)|^{r}(1+|m-n|)^{-(N+3)}
$$

by (5) and

$$
\sum_{|n| \leq 2^{k}}|f(n)|^{r}(1+|n|)^{-3} \leq C_{\delta_{0}}+C_{N}\left|\delta_{0}\right|^{r} \sum_{|n| \leq 2^{k+1}}|f(n)|^{r}(1+|n|)^{-3}
$$


by summing over $|n| \leq 2^{k}$ and integrating over $|\delta|<\delta_{0}<1$, where $\delta_{0}$ is chosen later and $C_{N}$ is independent of $\delta_{0}$. Denote $A_{k}=\sum_{2^{k-1}<|n| \leq 2^{k}}|f(n)|^{i}(1+|n|)^{-3}$. Therefore $\sum_{j=1}^{k} A_{j} \leq 2 C_{\delta_{0}}+$ $2 C_{N}\left|\delta_{0}\right|^{r} A_{k+1}$. Conversely if we assume $\sum_{j=1}^{k_{0}} A_{j} \geq 4 C_{\delta_{0}}$ for some $k_{0}$, then $\sum_{j=1}^{k_{0}+s} A_{j} \geq\left(1+\frac{1}{4 C_{N}\left|\delta_{0}\right|^{r}}\right)^{s} C_{\delta_{0}}$, which contradicts $\sum_{j=1}^{k_{0}}\left|A_{j}\right| \leq$ $C \sum_{j=1}^{2^{k_{0}+s}}(1+|n|)^{N} \leq C 2^{s N}$ provided $\delta_{0}$ is chosen small enough. This proved $\sum_{n \in Z}|f(n)|^{r}(1+|n|)^{-3}<+\infty$.

Furthermore by (5) we have

$$
|f(n)|^{r} \leq|f(n+\delta)|^{r}+C|\delta|^{r} \sum_{m \in Z}|f(m)|^{r}(1+|n-m|)^{-3}
$$

for some constant $C$ independent of $f$ and by integrating over $|\delta| \leq \delta_{0}$ for some sufficiently small $\delta_{0}>0$ we get

$$
\sum_{n}|f(n)|^{r}(1+|n|)^{-3} \leq C \int_{R}|f(x)|^{r}(1+|x|)^{-3} d x \leq C M\left(|f|^{r}\right)(0) .
$$

Therefore Lemma 1 is proved.

Lemma 2. Let the integer translates of the continuous function $\phi$ be globally linearly independent. If $m(x)=\sum_{n \in Z} C(n) \phi(x-n) \in M(p)$, then $\{C(n)\} \in l^{\infty}$ and $\|\{C(n)\}\|_{l^{\infty}(Z)} \leq C\|m\|_{M(p)}$.

Proof of Lemma 2: First we prove

$$
\|m\|_{L^{\infty}(R)} \leq C\|m\|_{M(p)}
$$

Obviously (6) is true when $1<p<\infty$ since Marcinkiewicz real interpolation, $\|m\|_{M(2)}=\|m\|_{L^{\infty}(R)}$ and $\|m\|_{M(p)}=\|m\|_{M\left(p^{\prime}\right)}$ where $p^{\prime}=\frac{p}{p-1}$. Hence the matter reduces to proving (6) for $0<p \leq 1$. For $f \in H^{p}(R)$, we have the atomic decomposition $f(x)=\sum_{k=0}^{\infty} \lambda_{k} a_{k}(x)$ with $C^{-1}\|f\|_{H^{p}(R)} \leq\left(\sum_{k=0}^{\infty}\left|\lambda_{k}\right|^{p}\right)^{\frac{1}{p}} \leq C\|f\|_{H^{p}(R)}$, where $a_{k}$ are $(p, 2, s)$ atoms and $s \geq \frac{1}{p}-1$. We call that $a$ is an $(p, 2, s)$ atom if there exists an interval $I$ such that $\operatorname{supp} a \subset I,\|a\|_{L^{2}(R)} \leq|I|^{\frac{1}{2}-\frac{1}{p}}$ and $\int x^{\alpha} a(x) d x=$ 0 for $0 \leq \alpha \leq s$. It is easy to show $\hat{a}(x)$ is continuous, $|\hat{a}(x)| \leq$ $C|I|^{1-\frac{1}{p}}$ and $|\hat{a}(x)| \leq C|x|^{s+1}|I|^{s+2-\frac{1}{p}}$. Hence $|\hat{a}(x)| \leq C|x|^{\frac{1}{p}-1}$ and $|\hat{f}(x)| \leq \sum_{k=0}^{\infty}\left|\lambda_{k}\right|\left|\hat{a}_{k}(x)\right| \leq C\left(\sum_{k=0}^{\infty}\left|\lambda_{k}\right|^{p}\right)^{\frac{1}{p}}|x|^{\frac{1}{p}-1}$. Denote $f_{t}(x)=$ $t^{-\frac{1}{p}} f\left(\frac{x}{t}\right)$. Therefore $f_{t}(x)=\sum_{k=0}^{\infty} \lambda_{k}\left(a_{k}\right)_{t}(x)$ and $C^{-1}\left(\sum_{k=0}^{\infty}\left|\lambda_{k}\right|^{p}\right)^{\frac{1}{p}} \leq$ 
$\left\|f_{t}\right\|_{H^{p}(R)} \leq C\left(\sum_{k=0}^{\infty}\left|\lambda_{k}\right|^{p}\right)^{\frac{1}{p}}$. Recall that $m \in M(p)$ for $0<p \leq 1$. Hence

$$
\left\|\left(m \hat{f}_{t}\right)^{\vee}\right\|_{H^{p}(R)} \leq C\left\|f_{t}\right\|_{H^{p}(R)} \leq C\|f\|_{H^{p}(R)}
$$

and

$$
\left|m(x) t^{\left(1-\frac{1}{p}\right)} \hat{f}(t x)\right| \leq C\|f\|_{H^{p}(R)}|x|^{\frac{1}{p}-1}
$$

for all $t>0$. Therefore $\left|m(x) \hat{f}\left(\frac{x}{|x|}\right)\right| \leq C\|f\|_{H^{p}(R)}$ for every $x \neq 0$ when we let $t=|x|^{-1}$ and (6) is proved for $0<p \leq 1$ by choosing $f \in H^{p}(R)$ such that $\hat{f} \equiv 1$ on the unit sphere.

Second we prove

$$
\|\{C(n)\}\|_{l^{\infty}(Z)} \leq C\|m\|_{L^{\infty}(R)} .
$$

By [1, Theorem 1.3], there exists a local algebraic dual $\left\{\Lambda_{n}\right\}$ of $\{\phi(x-$ $n)\}$, which says $\Lambda_{n} \phi(x-k)=\delta_{n k}$ and there exists a bounded set $K$ such that $\Lambda_{n} f=0$ when $f \in S(\phi)$ and $\operatorname{supp} f \cap(K+n)=\emptyset$, where we define the Kronekker symbol $\delta_{n k}$ by $\delta_{n n}=1$ and $\delta_{n k}=0$ when $n \neq k$. Recall that $\phi$ is continuous. Hence there exist finite points $x_{i} \in K$ and weights $C\left(x_{i}\right)$ such that $\Lambda_{n} f=\sum_{i} C\left(x_{i}\right) f\left(x_{i}+n\right)$ for every $f \in S(\phi)$. This shows $|C(n)|=\left|\Lambda_{n} m\right| \leq C\|m\|_{L^{\infty}}$ for every $n \in Z$. Therefore (7) holds and Lemma 2 is proved by combining (6) and (7).

Lemma 3. ([4] or [5, Theorem A.1]). Let $1<p<+\infty$ and $1<$ $q \leq+\infty$. Therefore the following Fefferman-Stein vector-valued maximal inequality

$$
\left\|\left(\sum_{k \in Z}\left|M f_{k}\right|^{q}\right)^{\frac{1}{q}}\right\|_{L^{p}(R)} \leq C\left\|\left(\sum_{k \in Z}\left|f_{k}\right|^{q}\right)^{\frac{1}{q}}\right\|_{L^{p}(R)}
$$

holds where $M$ denotes the Hardy-Littlewood maximal operator on $R$ as usual.

Let $h$ and $\eta$ be two Schwartz functions such that supp $\hat{\eta} \subset\left\{|x| \leq \frac{1}{20}\right\}$ and $\hat{\eta}(x)=1$ on $\left\{|x| \leq \frac{1}{40}\right\}$. Let $\Phi_{m}$ be as in the definition of $H^{p}(R)$. For $f \in H^{p}(T)$ we introduce a new type of paraproduct operator $P_{h}$ defined by

$$
P_{h} f(x)=\sum_{m \geq 0}\left(\eta_{m} * h\right)(x)\left(\Phi_{m} * f\right)(x),
$$

where $\eta_{m}(x)=2^{m} \eta\left(2^{m} x\right)$. 
Lemma 4. Let $P_{h}$ be defined by (8) and $h$ be a Schwartz function. Then $P_{h}$ maps $H^{p}(T)$ to $H^{p}(R)$,

$$
\left\|P_{h} f\right\|_{H^{p}(R)} \leq C\|f\|_{H^{p}(T)}
$$

Proof: Observe that $\left|\eta_{m} * h(x)\right| \leq C_{N}(1+|x|)^{-N}$ for every $N \geq 0$ and some $C_{N}$ independent of $m$. Also observe that $\operatorname{supp}\left(\left(\eta_{m} * h\right)\left(\Phi_{m} * f\right)\right)^{\wedge} \subset$ $\left\{\frac{11}{40} 2^{m} \leq|x| \leq \frac{19}{20} 2^{m}\right\}$ and $\Phi_{m} * f(x+k)=\Phi_{m} * f(x)$ for all $k \in Z$. Therefore

$$
\begin{aligned}
\left\|P_{h} f\right\|_{H^{p}(R)}^{p} & \leq C \int_{R}\left(\sum_{m \geq 0}\left|\left(\eta_{m} * h\right)(x)\left(\Phi_{m} * f\right)(x)\right|^{2}\right)^{\frac{p}{2}} d x \\
& \leq C \sum_{k \in Z} \int_{0}^{1}\left(\sum_{m \geq 0}(1+|k|)^{-\max \left(4, \frac{4}{p}\right)}\left|\Phi_{m} * f(x)\right|^{2}\right)^{\frac{p}{2}} d x \\
& \leq C\|f\|_{H^{p}(T)}^{p}
\end{aligned}
$$

and Lemma 4 is proved.

\section{Proof of theorems}

Proof of Theorem 1: Let $\{C(n)\}$ be a multiplier on $H^{p}(T)$ and supp $\phi \subset\left[-\frac{1}{4} M, \frac{1}{4} M\right]$ for some $M>1$. Denote $m(x)=\sum C(n) \phi(x-n)$. Let $\Phi_{k}$ be as in the definition of $H^{p}(R)$. Write $f=f_{0}+f_{1}+f_{2}$, where $f_{0}=\sum_{k \leq 2 M_{1}} \Phi_{k} * f$ and $f_{i}=\sum_{k \geq M_{1}} \Phi_{2 k+i} * f$ for $i=1,2$, where $M_{1}$ is a positive integer such that $2^{M_{1}} \geq 20 M$. Observe that

$$
m(x)\left(\Phi_{k} * f\right)^{\wedge}(x)=\sum_{|l| \leq 2^{2 M_{1}}+M} C(l) \phi(x-l)\left(\Phi_{k} * f\right)^{\wedge}(x)
$$

for $k \leq 2 M_{1}$. Write $\phi^{\vee}(x)=\sum_{n \in Z} \phi^{\vee}\left(\frac{n}{M}\right) \psi\left(x-\frac{n}{M}\right)$ for some $\psi \in$ $S(R)$ with supp $\hat{\psi} \subset\left\{|x| \leq \frac{3}{8}\right\}$, where $\phi^{\vee}$ denotes inverse Fourier trans- 
form. Therefore

$$
\begin{aligned}
& \int_{R}\left(\sum_{k \leq 2 M_{1}}\left|\left(m\left(\Phi_{k} * f\right)^{\wedge}\right)^{\vee}(x)\right|^{2}\right)^{\frac{p}{2}} d x \\
& \quad \leq C \sum_{|l| \leq 2^{2 M_{1}+M}}|C(l)|^{p}\left(\sum_{n \in Z}\left|\phi^{\vee}\left(\frac{n}{M}\right)\right|^{\min (p, 1)}\right)^{\max (p, 1)} \\
& \int_{R}\left(\sum_{k \leq 2 M_{1}}|\psi *|\left(\Phi_{k} * f\right)|(x)|^{2}\right)^{\frac{p}{2}} d x \\
& \quad \leq C\|\{C(l)\}\|_{l^{\infty}(Z)}^{p} \int_{R}\left(\sum_{k \leq 2 M_{1}}\left(M\left(\Phi_{k} * f\right)^{r}(x)\right)^{\frac{2}{r}}\right)^{\frac{p}{2}} d x \\
& \quad \leq C\|m\|_{\tilde{M}(p)}^{p}\|f\|_{H^{p}(R)}^{p},
\end{aligned}
$$

where $0<r<\min (p, 1)$. The first inequality follows Hölder inequality and $\sum_{n \in Z}\left|a_{n}\right| \leq\left(\sum_{n \in Z}\left|a_{n}\right|^{p}\right)^{\frac{1}{p}}$ for $0<p \leq 1$, the second inequality follows from Lemma 1 and

$$
\sup _{|y| \leq 2} \sum_{n \in Z}\left|\phi^{\vee}(y+n)\right|^{\min (p, 1)} \leq C \int|\hat{\phi}(x)|^{\min (p, 1)} d x
$$

(see [13, Lemma 6]), and the third inequality follows from Lemma 2 and Lemma 3. For $k \geq 2 M_{1}$, write $\phi(x)\left(\Phi_{k} * f\right)^{\wedge}(x+n)=$ $\sum_{l \in Z} C_{k, n}(l) e^{2 \pi i l x / M} \hat{\eta}(x / M)$ for some Schwartz function $\eta$ with supp $\hat{\eta} \subset\left\{|x| \leq \frac{3}{8}\right\}$. Therefore we get

$$
\left(m\left(\Phi_{k} * f\right)^{\wedge}\right)^{\vee}(x)=\sum_{l \in Z} \sum_{2^{k-2} \leq|n|<2^{k}} C(n) C_{k, n}(l) e^{-2 \pi i n x} \eta(M x-l)
$$

since $2^{M_{1}} \geq 20 M$ and

$$
\begin{aligned}
& \sum_{2^{k-2} \leq|n|<2^{k}} C_{k, n}(l) e^{-2 \pi i n x} \\
& =\int_{-\frac{1}{2}}^{\frac{1}{2}} \phi(y)\left(\sum_{n \in Z}\left(f * \Phi_{k}\right)^{\wedge}(y+n) e^{-2 \pi i n x}\right) e^{-2 \pi i l y} d y \\
& =\sum_{n \in Z}\left(f * \Phi_{k}\right)(x+n) \hat{\phi}\left(x+n-\frac{l}{M}\right)
\end{aligned}
$$


by Poisson summation formula. Hence

$$
\begin{aligned}
& \left\|\left(m \hat{f}_{i}\right)^{\vee}\right\|_{H^{p}(R)}^{p} \leq C \int_{R}\left(\sum_{k \geq M_{1}}\left|\left(m\left(\Phi_{2 k+i} * f\right)^{\wedge}\right)^{\vee}(x)\right|^{2}\right)^{\frac{p}{2}} d x \\
& \leq C \sum_{l \in Z} \int_{R}\left(\sum_{k \geq M_{1}}\left|\sum_{\substack{2^{2 k+i-2} \leq \\
|n|<2^{2 k+i}}} C(n) C_{2 k+i, n}(l) e^{-2 \pi i n x}\right|^{2}\right)^{\frac{p}{2}} \\
& \times(1+|M x-l|)^{-2} d x \\
& \leq C \sum_{l \in Z} \int_{-\frac{1}{2}}^{\frac{1}{2}}\left(\sum_{k \geq M_{1}}\left|\sum_{\substack{2^{2 k+i-2} \leq \\
|n|<2^{2 k+i}}} C(n) C_{2 k+i, n}(l) e^{-2 \pi i n x}\right|^{2}\right)^{\frac{p}{2}} d x \\
& \leq C\|\{C(n)\}\|_{\tilde{M}(p)}^{p} \sum_{l \in Z} \int_{-\frac{1}{2}}^{\frac{1}{2}}\left(\sum_{k \geq M_{1}}\left|\sum_{\substack{2 k+i-2 \\
2^{2 k \mid<2^{2 k+i}} \\
|n|<2^{2}}} C_{2 k+i, n}(l) e^{-2 \pi i n x}\right|^{2}\right)^{\frac{p}{2}} d x \\
& \leq C\|\{C(n)\}\|_{\tilde{M}(p)}^{p} \sum_{l \in Z} \int_{-\frac{1}{2}}^{\frac{1}{2}}\left(\sum _ { k \geq M _ { 1 } } \left(\sum_{n \in Z}\left|f * \Phi_{2 k+i}(x+n)\right|\right.\right. \\
& \left.\left.\times\left|\hat{\phi}\left(x+n-\frac{l}{M}\right)\right|\right)^{2}\right)^{\frac{p}{2}} d x \\
& \leq C\|\{C(n)\}\|_{\tilde{M}(p)}^{p}\left(\int|\hat{\phi}(x)|^{\min (p, 1)} d x\right)^{\max (p, 1)} \\
& \times\left(\sum_{n \in Z} \int_{-\frac{1}{2}}^{\frac{1}{2}}\left(\sum_{k \geq M_{1}}\left|f * \Phi_{2 k+i}(x+n)\right|^{2}\right)^{\frac{p}{2}} d x\right) \\
& \leq C\|\{C(n)\}\|_{\tilde{M}(p)}^{p}\|f\|_{H^{p}(R)}^{p},
\end{aligned}
$$

and Theorem 1 is proved.

Proof of Theorem 2: Let $m(x)=\sum_{n \in Z} C(n) \phi(x-n)$ be a multiplier of $H^{p}(R)$ and $f(x)=\sum_{l \in Z} \hat{f}(l) e^{2 \pi i l x}$ is a trigonometric polynomial. 
Observe that $|\hat{f}(k)| \leq C_{k}\|f\|_{H^{p}(T)}$. Therefore we assume $\hat{f}(0)=0$ without loss of generality. Write

$$
\begin{gathered}
=\sum_{n, k \in Z} \sum_{m \geq 0} C(n+k) \hat{f}(k) \hat{\Phi}\left(2^{-m} k\right) e^{2 \pi i k x} \int \hat{\eta}_{m}(\xi) \hat{h}(\xi) \phi(\xi-n) e^{-2 \pi i x \xi} d \xi \\
=\sum_{n \in Z} \sum_{m \geq 0} \Phi_{m} *\left(\tau_{n} f\right)(x) \int_{-\frac{1}{2}}^{\frac{1}{2}} e^{2 \pi i n y} \sum_{k \in Z} \hat{\phi}(y+k)\left(\eta_{m} * h-h\right)(x-y-k) d y \\
+\sum_{n \in Z}\left(\tau_{n} f\right)(x) \int_{-\frac{1}{2}}^{\frac{1}{2}} e^{2 \pi i n y} \sum_{k \in Z} \hat{\phi}(y+k) h(x-y-k) d y,
\end{gathered}
$$

where $\left(\tau_{n} f\right)(x)=\sum_{k \in Z} C(n+k) \hat{f}(k) e^{2 \pi i k x}$ and the second equality follows from Poission summation formula $\sum_{n \in Z} f(n)=\sum_{n \in Z} \hat{f}(n)$ and $\sum_{m \geq 0} \hat{\Phi}_{m}=1$ on $\{|\xi| \geq 1\}$. Let $h_{0}$ be a smooth function with compact support such that

$$
\sum_{k \in Z} \hat{\phi}(y+k) h_{0}(-y-k)=1 \text { on }\left[-\frac{1}{2}, \frac{1}{2}\right],
$$

since for every $y \in\left[-\frac{1}{2}, \frac{1}{2}\right]$ there exists $k \in Z$ for which $\hat{\phi}(y+k) \neq 0$ by the Fourier transform characterization of global linear independence of integer translates of $\phi[\mathbf{R}]$. Denote $h_{i}(x)=h_{0}\left(x-2^{-N} i\right)$ and the characteristic function on $\left[2^{-N} i, 2^{-N}(i+1)\right)$ by $\chi_{i}$, where $N$ is chosen later and $-2^{N-1} \leq i \leq 2^{N-1}-1$. Therefore multiplying $\chi_{i}$, on the two sides of (9), we get

$$
\begin{aligned}
& \left|\chi_{i}(x)\right|\left|\tau_{0} f(x)\right| \leq\left|\chi_{i}(x)\left(m\left(P_{h_{i}} f\right)^{\wedge}\right)^{\vee}(x)\right| \\
& +C \sum_{n \in Z} \chi_{i}(x)\left|\tau_{n} f(x)\right| 2^{-\frac{N}{2}}(1+|n|)^{-2 / \min (p, 1)} \\
& +C A_{m_{0}}\left|\chi_{i}(x)\right| \sum_{n \in Z}(1+|n|)^{-2 / \min (p, 1)}\left(\sum_{m \geq 0}\left|\Phi_{m} *\left(\tau_{n} f\right)(x)\right|^{2}\right)^{\frac{1}{2}},
\end{aligned}
$$

where $A_{m_{0}}(h)=\sup _{-2^{N-1} \leq i \leq 2^{N}-1} \sum_{|k| \leq \frac{2}{p}+2}\left(\sum_{m \geq m_{0}} \| \eta_{m} *\left(\frac{\partial}{\partial x}\right)^{k} h_{i}-\right.$ $\left.\left(\frac{\partial}{\partial x}\right)^{k} h_{i} \|_{L^{\infty}}^{2}\right)^{\frac{1}{2}}$ and $\hat{f}(k)=0$ for $|k| \leq 2^{m_{0}}$. It is easy to prove $A_{m_{0}}(h) \leq$ $C 2^{-m_{0}}$. Recall that (8), $m \in M(p)$ and $|\hat{f}(k)| \leq C_{k}\|f\|_{H^{p}(T)}$ for $|k| \leq$ $2^{m_{0}}$. Hence

$$
\begin{aligned}
\left\|\tau_{0} f\right\|_{L^{p}(T)}^{p} \leq C_{N, m_{0}}\|m\|_{M(p)}^{p}\|f\|_{H^{p}(T)}^{p} & \\
& +C\left(2^{-N / 2}+2^{-m_{0}}\right) \sum_{n \in Z}\left\|\tau_{n} f\right\|_{H^{p}(T)}^{p}(1+|n|)^{-2}
\end{aligned}
$$


holds for some constant $C$ independent of $N$ and $m_{0}$ by Lemma 4 . Define the Hilbert transform $H$ by

$$
H f(x)=\sum_{k>0} f_{k} e^{2 \pi i k x}-\sum_{k<0} f_{k} e^{2 \pi i k x}
$$

for $f(x)=\sum_{k \in Z} f_{k} e^{2 \pi i k x}$. Therefore $H$ maps $H^{p}(T)$ to $H^{p}(T)$ and

$$
\|f\|_{H^{p}(T)} \leq C\|H f\|_{L^{p}(T)}+C\|f\|_{L^{p}(T)} .
$$

In high spatial dimensions, we can use Riesz transforms to replace Hilbert transform [8]. Hence we have

$$
\begin{aligned}
\left\|\tau_{0} f\right\|_{H^{p}(T)}^{p} \leq C_{N, m_{0}}\|m\|_{M(p)}^{p}\|f\|_{H^{p}(T)}^{p} & \\
& +C\left(2^{-N / 2}+2^{-m_{0}}\right) \sum_{n \in Z}\left\|\tau_{n} f\right\|_{H^{p}(T)}^{p}(1+|n|)^{-2}
\end{aligned}
$$

Observe that $\sup _{n \in Z}\left\|\tau_{n} f\right\|_{H^{p}(T)}<+\infty$ when $f$ is a trigonometric polynomial and $\{C(n)\} \in l^{\infty}$ by Lemma 2 . Therefore by choosing $N$ and $m_{0}$ large enough we get

$$
\sup _{n \in Z}\left\|\tau_{n} f\right\|_{H^{p}(T)} \leq C\|m\|_{M(p)}^{p}\|f\|_{H^{p}(T)}
$$

for every trigonometric polynomial $f$. Theorem 2 is proved.

Proof of Theorem 3: First the right inequality. Since supp $\hat{f} \subset$ $\left[-\frac{1}{2}+\varepsilon, \frac{1}{2}-\varepsilon\right]$ for some $0<\varepsilon<\frac{1}{2}$. Write $f(x)=\sum_{n \in Z} f(n) \psi(x-n)$ where $\psi$ is a Schwartz function such that $\hat{\psi}(x)=1$ on $\left[-\frac{1}{2}+\frac{\epsilon}{4}, \frac{1}{2}-\frac{\epsilon}{4}\right]$ and $\operatorname{supp} \hat{\psi} \subset\left[-\frac{1}{2}+\frac{\epsilon}{8}, \frac{1}{2}-\frac{\epsilon}{8}\right]$. Observe that

$$
\begin{aligned}
\Phi_{m} * f(x) & =\sum_{n \in Z} f(n) \int \hat{\Phi}\left(2^{-m} \xi\right) \overline{\hat{\psi}}(\xi) e^{-i 2 \pi(x-n) \xi} d \xi \\
& =\int\left(\varphi_{-m}^{*} f\right)^{\wedge}(\xi) e^{-2 \pi i x \xi} d \xi
\end{aligned}
$$

where we denote $\left(\varphi_{m}^{*} f\right)^{\wedge}(\xi)=\hat{\Phi}\left(2^{m} \xi\right) \overline{\hat{\psi}}(\xi) \hat{f}(\xi)$ for $m \in Z$ and $\hat{f}(\xi)=$ $\sum_{n \in Z} f(n) e^{2 \pi i n \xi}$. Observe that

$$
\Phi_{m} * f(x)=\sum_{n \in Z} \varphi_{-m}^{*}(f)(n) g(x-n)
$$


where $g$ is a Schwartz function with supp $\hat{g} \subset\left[-\frac{1}{2}+\frac{\xi}{16}, \frac{1}{2}-\frac{\xi}{16}\right]$ when $m \leq 0, \Phi_{m} * f=0$ when $m \geq 1$ and $\Phi$ is chosen approciately. Therefore by Lemma 1 we get

$$
\begin{aligned}
\left\|\left(\sum_{m \in Z}\left|\Phi_{m} * f(x)\right|^{2}\right)^{\frac{1}{2}}\right\|_{L^{p}(R)} & \leq\left\|\left(\sum_{m \geq 0}\left(\sum_{n \in Z} \varphi_{m}^{*}(f)(n) g(x-n)\right)^{2}\right)^{\frac{1}{2}}\right\|_{L^{p}(R)} \\
& \leq C\left\|\left(\sum_{m \geq 0}\left|\varphi_{m}^{*}(f)(n)\right|^{2}\right)^{\frac{1}{2}}\right\|_{L^{p}(Z)} \\
& \leq C\|\{f(n)\}\|_{H^{p}(Z)} .
\end{aligned}
$$

Now the left inequality. By the procedure used as in the proof of Lemma 1, it suffices to show $\sum_{k}\left(\sum_{m \leq 0}\left|\tilde{\Phi}_{m} * f(k)\right|^{2}\right)^{\frac{p}{2}}<+\infty$. Since $f \in$ $S^{\prime}(R)$, we get $\left|\tilde{\Phi}_{m} * f(k)\right| \leq C_{N, m}\left(2^{-m}+|k|\right)^{N}$ for some $N$ and all $m \geq 0$. As in the proof of Lemma 1, we get $\sum_{k \in Z}\left|\tilde{\Phi}_{m} * f(k)\right|(1+|k|)^{-N_{1}}<+\infty$ and $\sum_{k \in Z}\left|\tilde{\Phi}_{m} * f(k)\right|(1+|k|)^{-N_{1}} \leq C \sum_{k \in Z}\left|\tilde{\Phi}_{m} * f(k+\delta)\right|(1+|k+\delta|)^{-N_{1}}$ for some $C$ independent of $f$ and $m$, where $N_{1}$ are chosen later. Therefore

$$
\left(\sum_{m \geq 0}\left|\tilde{\Phi}_{m} * f(k)\right|^{2}\right)^{\frac{p}{2}} \leq C \int\left(\sum_{m \geq 0}\left|\tilde{\Phi}_{m} * f(x)\right|^{2}\right)^{\frac{p}{2}} d x(1+|k|)^{N_{1}}
$$

for some $N_{1} \leq \frac{2}{p}+2$. Still by the procedure used as in the proof of Lemma 2, we get $\sum_{k \in Z}\left(\sum_{m \geq 0}\left|\tilde{\Phi}_{m} * f(k)\right|^{2}\right)^{\frac{p}{2}}<+\infty$ and $\sum_{k \in Z}\left(\sum_{m \geq 0}\left|\tilde{\Phi}_{m} * f(k)\right|^{2}\right)^{\frac{p}{2}} \leq C\|f\|_{H^{p}(R)}^{2}$. Hence the left inequality and Theorem 3 is proved.

\section{References}

1. A. Ben-ARtzi AND A. Ron, On the integer translates of a compactly supported function: dual bases and linear projections, SIAM J. Math. Anal. 21 (1990), 1550-1562.

2. E. Berkson And T. A. Gillesprie, Transference and extension of Fourier multipliers for $L^{p}(T)$, J. London Math. Soc. 41 (1990), 472-488.

3. R. E. Edwards AND G. I. GUNDRY, "Littlewood-Paley and multiplier theory," Springer-Verlag, Berlin, 1977. 
4. C. Fefferman and E. M. Stein, Some maximal inequalities, Amer. J. Math. 93 (1971), 107-115.

5. M. Frazier and B. JaWerth, A discrete transform and decomposition of distribution spaces, J. Funct. Anal. 93 (1990), 34-170.

6. M. Jodeit, Restriction and extension of Fourier multipliers, Studia Math. 34 (1970), 215-226.

7. K. DE LeEuw, On $L^{p}$ multilpliers, Ann. of Math. 81 (1965), 364-379.

8. Z-X LiU, Multipliers on real Hardy spaces, Sci. Sinica 35 (1992), $55-69$.

9. A. RoN, A necessary and sufficient condition for the linear independence of the integer translates of a compactly supported distribution, Constr. Approx. 5 (1989), 297-308.

10. C. E. Shannon, Communication in the presence of noise, Proc. of the IRE 37 (1949), 10-21.

11. P. SJöLIN, Convolution with oscillating kernels in $H^{p}$ spaces, $J$. London Math. Soc. 23 (1981), 442-454.

12. E. M. Stein, M. H. Taibleson and G. Weiss, Weak type estimates for maximal operators on certain $H^{p}$ spaces, Rend. Circ. Mat. Palermo, supplemente 1 (1981), 81-97.

13. Q. sun, Sequence spaces and stability of integer translates, Zeit. Anal. Anwendungen 12 (1993), 567-584.

14. R. TORRES, Space of sequences, sampling theorem and functions of exponential type, Studia Math. 100 (1991), 51-74.

15. H. TRIEBEL, "Theory of function spaces," Akad. Verlagsges, Leipzig, 1983.

Center for Mathematical Sciences

Zhejiang University

Hangzhou Zhejiang 310028

P. R. OF CHINA

Rebut el 26 d'Abril de 1994 